\title{
Hawking radiation Schwarzschild black hole in fuzzy space
}

\author{
A. EL Boukili*, M. Nach, M. B. Sedra \\ Laboratoire des Hautes énergies, Sciences de l'Ingénierie et Réacteurs, \\ Université Ibn Tofail, Faculté des Sciences, Département de Physique, \\ Kénitra, Morocco \\ *Corresponding author E-mail:aelboukili@gmail.com
}

\begin{abstract}
Given the importance of Hawking radiation in the areas of black holes, we present in this paper an overview about the fuzzy black hole and its thermodynamic properties. We introduce the Hawking radiation of this class of black holes via complex path method and we give the possibility to estimate the evaporation time of the Schwarzschild black hole in fuzzy space.
\end{abstract}

Keywords: Complex path method, evaporation time, fuzzy space, Hawking radiation, Schwarzschild black hole.

\section{Introduction}

Hawking radiation is a theoretical prediction made by the British physicist Stephen Hawking, which gives the thermal properties of the black hole [1]. In 1974, the work of Stephen Hawking on theoretical model clarifies how the black hole can radiate black body [2]. However, Hawking radiation can be viewed in different types of black hole namely charged rotating black holes [3], Kerr-Newman black hole [4], BTZ black hole [5], Schwarzschild black hole [6], Reissner-Nordstrom black hole [7] and AdS Schwarzschild black hole [8].

In the same context, the black hole evaporation produces a more consistent view of black hole thermodynamics, by showing how black holes interact thermally with the rest of the universe [9].

In this paper, we study Hawking radiation and evaporation time of black hole in a fuzzy space [10], such space represent another type of non commutative geometry, which appear naturally in the string/M theory. It is also known to correspond to the sphere D2-branes in string theory with background linear B-field [11]. Therefore, it is interesting to see how the fuzzy space will modify the black hole properties of Schwarzschild Black hole.

The paper is organized as follows. In Sec. 2, we review the main features of Fuzzy Schwarzschild Black hole. In Sec. 3 , we study the Hawking radiation of Black hole in fuzzy space via complex path analysis. In Sec. 4, we will gives the estimated evaporation time of the geometry of fuzzy black hole. A brief conclusion is reported in Sec. 5 .

\section{Fuzzy Schwarzschild black hole}

We consider Schwarzschild Black hole in fuzzy space with the Mass density of point particle described by [12]

$$
\rho_{h}(r)=\frac{1}{2 \pi h^{2}} \exp \left(-\frac{r}{h}\right)
$$

The covariant conservation law $\nabla_{\mu} T^{\mu v}=0 \quad$ and the condition of the metric coefficient $\quad g_{00}=-g_{r r}^{-1}$ for the non commutative Schwarzschild like metric, the energy momentum tensor gives by

$$
T_{v}^{\mu}=\operatorname{Diag}\left(-\rho_{h}(r), p_{r}(r), p_{\perp}(r), p_{\perp}(r)\right)
$$

with 


$$
p_{r}=-\rho_{h}, \quad p_{\perp}=p_{r}-\frac{r}{2} \partial_{r} \rho_{h}
$$

The asymptotic solution of Einstein equation (2), using (1) as the matter source, is the same as replacing the mass of Dirac delta function source in Schwarzschild space-time by the effective mass of smeared source

$$
\begin{aligned}
M_{h}(r) & =M \int d^{3} x \rho_{h}(r)=4 \pi \mathrm{M} \int_{0}^{\mathrm{r}} r^{\prime 2} \rho_{h}\left(r^{\prime}\right) d r^{\prime} \\
& =M\left[1-\left(\frac{1}{2}\left(\frac{r}{h}\right)^{2}+\frac{r}{h}+1\right) \exp \left(-\frac{r}{h}\right)\right]
\end{aligned}
$$

The geometry of Fuzzy black hole is described by the line element

$$
d S^{2}=-\left(1-\frac{2 M_{h}(r)}{r}\right) d t^{2}+\left(1-\frac{2 M_{h}(r)}{r}\right)^{-1} d r^{2}+r^{2}\left(d \theta^{2}+\sin ^{2} \theta d \varphi^{2}\right)
$$

Where

$$
g_{00}(r)=g^{r r}(r)=f(r)=1-\frac{2 M_{h}(r)}{r}
$$

The event horizon of the black hole gives by setting $f\left(r_{h}\right)=0$, the nonzero solution of this equation satisfies

$$
r_{h}=2 M_{h}(r)=2 M\left[1-\left(\frac{1}{2}\left(\frac{r_{h}}{h}\right)^{2}+\frac{r_{h}}{h}+1\right) \exp \left(-\frac{r_{h}}{h}\right)\right]
$$

This equation cannot be solved in closed form. However, by plotting $\mathrm{f}(\mathrm{r})$ one can read intersections with the r-axis and determine numerically the existence of horizon(s) and their radii:

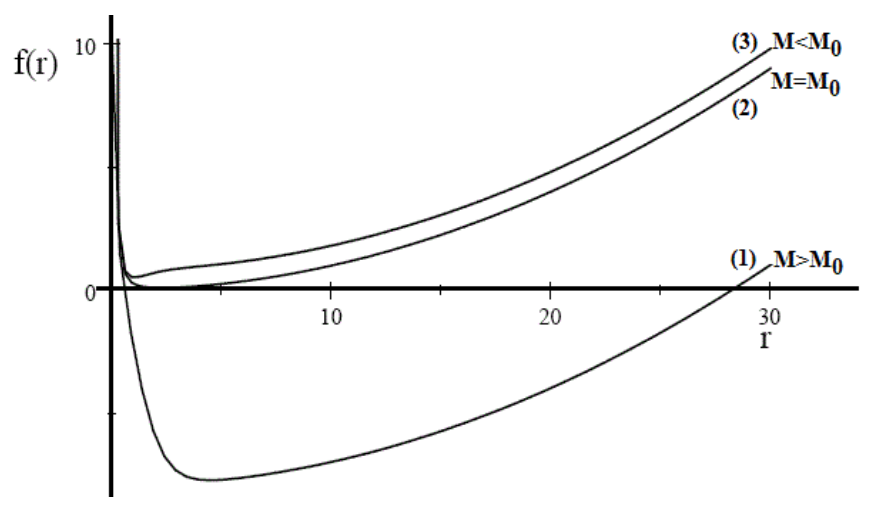

Figure1: Metric function $f$ according to $\mathrm{r}$. We have taken the values ( $h=0.1$ and $M_{0} \approx 0.001$ ).

This figure shows that the existence of the Mass M introduces new behavior with respect to the non commutative black hole studied by Myung and Yoon [13]. Instead of a single-event horizon, there are different possibilities: (i) We have two distinct horizons for $M>M_{0}$, (ii) In this case we have one degenerate horizon (external black hole) for $M=M_{0}$, (iii) No horizon for $M<M_{0}$.

The Hawking temperature is calculated by 


$$
T_{H}=\left(\frac{1}{4 \pi} \frac{d g_{00}}{d r}\right)_{r=r_{h}}=\frac{1}{4 \pi r_{h}}\left[1-\frac{\frac{1}{2}\left(\frac{r_{h}}{h}\right)^{3} \exp \left(-\frac{r_{h}}{h}\right)}{1-\left(\frac{1}{2}\left(\frac{r_{h}}{h}\right)^{2}+\frac{r_{h}}{h}+1\right) \exp \left(-\frac{r_{h}}{h}\right)}\right]
$$

\section{Hawking radiation in fuzzy space}

We consider a (1+1)-dimensional spacetime which has the line element

$$
d S^{2}=-f(r) d t^{2}+f^{-1}(r) d r^{2}
$$

where $f(r)$ is an arbitrary function of $r$. We use the method of complex path analysis for examine of the Hawking radiation [14]. The function $f(r)$ vanishes at $r_{0}$ and $f^{\prime}(r)$ is nonzero at $r_{0}$. We expand $f(r)$ around the point $r_{0}$ gives

$$
\begin{aligned}
f(r) & =f\left(r_{0}\right)+f^{\prime}\left(r_{0}\right)\left(r-r_{0}\right)+\mathrm{O}\left[\left(r-r_{0}\right)^{2}\right] \\
& =f^{\prime}\left(r_{0}\right)\left(r-r_{0}\right)+\mathrm{O}\left[\left(r-r_{0}\right)^{2}\right] \\
& =\mathbf{R}\left(r_{0}\right)\left(r-r_{0}\right)
\end{aligned}
$$

For the fuzzy black hole we have

$$
\begin{aligned}
f(r) & =\left(1-\frac{2 M_{h}\left(r_{0}\right)}{r}\right) \\
& =\left(2 M_{h}\left(r_{0}\right)\right)^{-1}\left(r-2 M_{h}\left(r_{0}\right)\right)+o\left[\left(r-2 M_{h}\left(r_{0}\right)\right)^{2}\right]
\end{aligned}
$$

with $\mathbf{R}\left(r_{0}\right)=\left(2 M_{h}\left(r_{0}\right)\right)^{-1}, r_{0}=2 M_{h}\left(r_{0}\right)$ and $\mathbf{R}\left(r_{0}\right) \neq 0$.

The Klein-Gordon equation satisfied by the scalar field is

$$
\left(\square+\frac{m_{0}^{2}}{\hbar^{2}}\right) \Phi=0
$$

where the operator $\square$ is to be evaluated using metric (9). In the background of (9) the last equation can be written by

$$
-\frac{1}{f(r)} \frac{\partial^{2} \Phi}{\partial t^{2}}+\frac{\partial}{\partial r}\left(f(r) \frac{\partial \Phi}{\partial r}\right)=-\frac{m_{0}^{2}}{\eta^{2}} \Phi
$$

The semi-classical wave functions satisfying the above are obtained by making the standard ansatz

$$
\phi(x, t)=\exp \left(\frac{i}{\eta} \mathbf{S}(x, t)\right)
$$

Substituting this ansatz into equation (13) we obtain

$$
-\frac{1}{f(r)}\left(\frac{\partial \mathbf{s}}{\partial t}\right)^{2}+f(r)\left(\frac{\partial \mathbf{s}}{\partial r}\right)^{2}-m_{0}^{2}+\frac{i}{\eta}\left[\frac{1}{f(r)} \frac{\partial^{2} \mathbf{s}}{\partial t^{2}}-f(r) \frac{\partial^{2} \mathbf{s}}{\partial r^{2}}-\frac{d f(r)}{d r} \frac{\partial \mathbf{s}}{\partial r}\right]
$$

Expanding $\mathbf{S}$ in a power series of $\eta / i$ : 


$$
\mathbf{S}(r, t)=\sum_{n=0}\left(\frac{\eta}{i}\right)^{n} \mathbf{S}_{n}(r, t)
$$

Neglecting terms of hight order in $\eta / i, \mathrm{~S}_{0}$ gives rise to

$$
-\frac{1}{f(r)}\left(\frac{\partial \mathbf{S}_{0}}{\partial t}\right)^{2}+f(r)\left(\frac{\partial \mathbf{S}_{0}}{\partial r}\right)^{2}-m_{0}^{2}=0
$$

and the solution is given by

$$
\mathbf{S}_{0}=-\mathbf{E} t \pm \int^{r} \frac{d r}{f(r)} \sqrt{\mathbf{E}^{2}-m_{0}^{2} f(r)}
$$

here $\mathbf{E}$ is a constant which is identified to energy. To simplify, we take $m_{0}=0$. The semi-classical propagator $\mathbf{K}\left(t_{1}, r_{1}, t_{2}, r_{2}\right)$ for a particle propagating from a space-time point $\left(t_{1}, r_{1}\right)$ to $\left(t_{2}, r_{2}\right)$ in the saddle point is given by

$$
\mathbf{K}\left(t_{1}, r_{1}, t_{2}, r_{2}\right)=\mathbf{N} \exp \left[\frac{i}{\eta} \mathbf{S}_{0}\left(t_{1}, r_{1}, t_{2}, r_{2}\right)\right]
$$

with $\mathbf{N}$ is a normalization constant and $\mathbf{S}_{0}$ is given by

$$
\mathbf{S}_{0}\left(t_{1}, r_{1}, t_{2}, r_{2}\right)=-\mathbf{E}\left(t_{2}-t_{1}\right) \pm \mathbf{E} \int_{x 1}^{x 2} \frac{d r}{f(r)}
$$

Consider an outgoing particle at $r=r_{1}<r_{0}$, the modulus square of the amplitude for this particle to cross the horizon gives the probability of emission of the particle. The contribution to $S_{0}$ in the ranges $\left(r_{1}, r_{0}-\varepsilon\right)$ and $\left(r_{0}+\varepsilon, r_{2}\right)$ is real. We take the contour to lie in the upper complex plane and find:

\section{Emission case:}

$$
\begin{aligned}
\mathbf{S}_{0-\text { Emission }} & =-\mathbf{E} \lim _{\varepsilon \rightarrow 0} \int_{r_{0}-\varepsilon}^{r_{0}+\varepsilon} \frac{d r}{f(r)}+\text { real part } \\
& =\frac{i \pi \mathbf{E}}{2 M}\left[1-\left(\frac{1}{2}\left(\frac{r_{0}}{h}\right)^{2}+\frac{r_{0}}{h}+1\right) \exp \left(-\frac{r_{0}}{h}\right)\right]^{-1}+\text { real part }
\end{aligned}
$$

\section{Absorption case:}

$$
\begin{aligned}
\mathbf{S}_{0-\text { Absorption }} & =-\mathbf{E} \lim _{\varepsilon \rightarrow 0} \int_{r_{0}+\varepsilon}^{r_{0}-\varepsilon} \frac{d r}{f(r)}+\text { real part } \\
& =-\frac{i \pi \mathbf{E}}{2 M}\left[1-\left(\frac{1}{2}\left(\frac{r_{0}}{h}\right)^{2}+\frac{r_{0}}{h}+1\right) \exp \left(-\frac{r_{0}}{h}\right)\right]^{-1}+\text { real part }
\end{aligned}
$$

with $\mathbf{S}_{0-\text { Emission }}$ and $\mathbf{S}_{0-\text { Absorption }}$ are respectively the emission and the absorption function. Taking the modulus Square to get the probability gives,

$$
P_{\mathbf{0} \text {-Emission }} \propto \exp \left[\frac{-2 \pi \mathbf{E}}{\mathbf{R}\left(r_{0}\right)}\right], P_{\mathbf{0} \text {-Absorption }} \propto \exp \left[\frac{2 \pi \mathbf{E}}{\mathbf{R}\left(r_{0}\right)}\right]
$$


So that

$$
P_{0 \text {-Emission }}=\exp \left[\frac{-4 \pi \mathbf{E}}{\mathbf{R}\left(r_{0}\right)}\right] P_{\mathbf{0} \text {-Absorption }}
$$

The temperature $\beta^{-1}$ the absorption and emission probabilities are related,

$$
P_{\text {0-Emission }}=\exp (-\beta \mathbf{E}) P_{\mathbf{0} \text {-Absorption }}
$$

we obtain the expression of the Hawking temperature of 2D fuzzy Schwarzschild black hole is,

$$
\begin{aligned}
T_{H} & =\beta^{-1}=\frac{\eta}{4 \pi}\left|\mathbf{R}\left(r_{0}\right)\right| \\
T_{H} & =\beta^{-1}=\frac{\eta}{8 \pi}\left(M_{h}\left(r_{0}\right)\right)^{-1} \\
& =\frac{\eta}{8 \pi}\left[M\left[1-\left(\frac{1}{2}\left(\frac{r_{0}}{h}\right)^{2}+\frac{r_{0}}{h}+1\right) \exp \left(-\frac{r_{0}}{h}\right)\right]\right]^{-1} \\
& =\frac{\eta}{8 \pi M}\left[1-\left(\frac{1}{2}\left(\frac{r_{0}}{h}\right)^{2}+\frac{r_{0}}{h}+1\right) \exp \left(-\frac{r_{0}}{h}\right)\right]^{-1}
\end{aligned}
$$

\section{Black hole evaporation}

The black hole evaporation time produces a more consistent view of black hole thermodynamics, by showing how black holes interact thermally with the rest of the universe. In Fuzzy space the Stefan--Boltzmann--Schwarzschild--Hawking black hole radiation power law derivation is given by [15]

$$
\mathrm{P}=-\frac{d M}{d t}=T_{H}^{2}=\frac{\eta^{2}}{64 \pi^{2} M^{2}}\left[1-\left(\frac{1}{2}\left(\frac{r}{h}\right)^{2}+\frac{r}{h}+1\right) \exp \left(-\frac{r}{h}\right)\right]^{-2}
$$

Using the power $P$ it is possible to estimate the evaporation time of the geometry of fuzzy black hole as follows

$$
t_{\text {evap }}=\frac{M}{|d M / d t|}=\frac{64 \pi^{2} M^{3}}{\eta^{2}}\left[1-\left(\frac{1}{2}\left(\frac{r}{h}\right)^{2}+\frac{r}{h}+1\right) \exp \left(-\frac{r}{h}\right)\right]^{2}
$$

here $\mathrm{h}$ is the parameter of fuzzy space and $\eta=6.6260755 \times 10^{-34} \mathrm{~m}^{2} \mathrm{~kg} / \mathrm{s}$ is the Planck's constant. From the equation (29), we note that the parameter $\mathrm{h}$ is involved in the expression of evaporation time as follows: When $h \rightarrow 0$, the evaporation time approaches to a standard case of Schwarzschild black hole ( $t_{\text {evap }}=\frac{4 \times 64 \pi^{2} M^{3}}{\eta^{2}}$ ), but when $h \rightarrow \infty$ the evaporation time approaches to 0 strongly.

\section{Conclusion}

In this work, we recalled some basic concept of Schwarzschild Black Hole in fuzzy space and their Thermodynamics properties. However, a main goal of this paper is to study the Hawking radiation and the evaporation time estimated of fuzzy Schwarzschild black hole by using the method of complex paths developed in [14].

We have to underline that the relevance of our work can be related also to the importance of the complex paths method. 


\section{References}

[1] D. N. Page," Hawking radiation and black hole thermodynamics", New J. Phys., 7, (2005), 203.

[2] S. W. Hawking, " Breakdown of predictability in gravitational collapse", Phys. Rev. D, 14, (1976), 2460;

M.K.Parikh and F.Wilczek, " Hawking Radiation As Tunneling ”, Phys. Rev. Lett., 85, (2000), 5042.

[3] Keiju Murata and Jiro Soda, "Hawking radiation from rotating black holes and gravitational anomalies ", Phys. Rev. D, 74, (2006), 044018.

[4] Shuang-Qing Wu and Jun-Jin Peng, " Thermodynamics and Hawking radiation of five-dimensional rotating charged Gödel black holes ", Phys. Rev. D, 83, (2011), 044028.

[5] Qing-Quan Jiang, Shuang-Qing Wu and Xu Cai, ” Hawking radiation from (2+1)-dimensional BTZ black holes ”, Phys. Lett. B, 651, (2007), 58-64.

[6] Sumit R. Das, Samir D. Mathur and P. Ramadevi, " Hawking radiation from four-dimensional Schwarzschild black holes in M theory ", Phys . Rev. D, 59, (1999), 084001.

[7] Sunandan Gangopadhyay, "Hawking radiation from a Reissner-Nordström black hole with a global monopole via covariant anomalies and effective action ", Phys. Rev. D, 78, (2008), 044026

[8] Jun-Jin Peng and Shuang-Qing Wu," Hawking radiation of black holes in infrared modified Hořava-Lifshitz gravity ", Eur. Phys. J. C, 66, (2010), 325-331.

[9] Elisabetta Di Grezia, Giampiero Esposito and Gennaro Miele," Black hole evaporation in a spherically symmetric non-commutative spacetime", J. Phys. A: Math. Theor. 41, (2008), 164063;

M. Angheben, M. Nadalini, L. Vanzo and S. Zerbini, "Hawking radiation as tunneling for extremal and rotating black holes ", JHEP, 05, (2005), 014

[10] Wung-Hong Huang, " Thermodynamics on Fuzzy Spacetime ”, JHEP, 0908, (2009), 102.

[11] Tom Banks and John Kehayias, "Fuzzy geometry via the spinor bundle, with applications to holographic space-time and matrix theory ", Phys. Rev. D, 84, (2011), 086008.

[12] Piero Nicolini, Anais Smailagic and Euro Spallucci, " Noncommutative geometry inspired Schwarzschild black hole ", Phys. Lett. B, 632, (2006), 547-551.

[13] Y S Myung and M Yoon,” Regular black hole in three dimensions ”, Eur. Phys. J. C, 62, (2009), 405-411.

[14] K. Srinivasan and T. Padmanabhan, " Particle production and complex path analysis ", Phys. Rev. D, 60, (1999), 024007.

[15] S. W. Hawking, " Evaporation of two-dimensional black holes ”, Phys. Rev. Lett., 69, (1992), 406-409. 